\title{
L858R-positive lung adenocarcinoma with KRAS G12V, EGFR T790M and EGFR L858R mutations: A case report
}

\author{
XIANHONG XIANG ${ }^{1 *}$, JIANXING YU $^{2 *}$, YINGRONG LAI ${ }^{3 *}$, WEILING HE ${ }^{4}$, \\ SHUHUA $\mathrm{LI}^{3}$, LIANTANG WANG ${ }^{3}$ and ZUNFU $\mathrm{KE}^{3}$ \\ Departments of ${ }^{1}$ Interventional Radiology, ${ }^{2}$ Private Medical Services and Healthcare Center, ${ }^{3}$ Pathology and \\ ${ }^{4}$ Gastrointestinal Surgery, The First Affiliated Hospital of Sun Yat-Sen University, Guangzhou, Guangdong 510080, P.R. China
}

Received August 17, 2014; Accepted May 15, 2015

DOI: $10.3892 / \mathrm{ol} .2015 .3435$

\begin{abstract}
Improvement in the current understanding of the molecular basis of lung cancer at multiple levels, including the genetic, epigenetic and protein levels, has the potential to impact the diagnosis, prognosis and treatment of lung cancer. The mutation status of the tyrosine kinase domain of epidermal growth factor receptor (EGFR) is known to be a predictor of the response to gefitinib in lung cancer. Furthermore, mutations in the EGFR and KRAS genes appear to be mutually exclusive. The present study reports a rare case of a patient harboring two EGFR mutations (L858R and T790M) and a KRAS mutation (G12V). The development of gefitinib resistance was detected in the subsequent treatment. The present study indicates that EGFR and KRAS mutational analysis should be recommended for all patients with non-small-cell lung carcinoma.
\end{abstract}

\section{Introduction}

Mutations in the epidermal growth factor receptor (EGFR) gene are closely associated with the response of cells to EGFR tyrosine kinase inhibitors (TKIs), including erlotinib and gefitinib, particularly in lung adenocarcinoma. The incidence

Correspondence to: Professor Liantang Wang or Dr Zunfu Ke, Department of Pathology, The First Affiliated Hospital of Sun Yat-Sen University, 58 Zhongshan Road II, Guangzhou, Guangdong 510080, P.R. China

E-mail: wanglt@mail.sysu.edu.cn

E-mail: kezunfu@126.com

${ }^{*}$ Contributed equally

Abbreviations: KRAS, kirsten rat sarcoma viral oncogene homolog; EGFR, epidermal growth factor receptor; TKI, tyrosine kinase inhibitor; NSCLC, non-small cell lung cancer; CT, computed tomography; IHC, immunohistochemical; CEA, carcinoembryonic antigen

Key words: adenocarcinoma, epidermal growth factor receptor, mutation, non-small cell lung cancer of resistance to EGFR TKIs in patients with non-small-cell lung carcinoma (NSCLC) is $10-20 \%$ (1). Furthermore, clinical trials have revealed that oral EGFR TKIs may lead to increased response rates and progression-free survival rates compared with standard chemotherapies in patients with $\operatorname{NSCLC}(2,3)$. However, numerous patients with NSCLC eventually develop resistance to these EGFR inhibitors. The T790M mutation in exon 20 of the EGFR gene has been reported as one of the driving mutations for the acquired resistance to gefitinib (4). Approximately one-half of the patients that acquire resistance to gefitinib are found to harbor the T790M EGFR mutation (5). In addition, 15-20\% of NSCLC tumors harbor mutations in exon 2 of the Kirsten rat sarcoma viral oncogene homolog (KRAS) gene (6-8). As a prognostic marker, KRAS mutations in resected NSCLC tissues have been found to be associated with a shorter overall survival time compared with patients with EGFR mutations. Although numerous current studies suggest that EGFR and KRAS mutations are mutually exclusive (9-11), as a downstream signal molecule of the EGFR pathway, a mutation in KRAS may be a predictor for primary resistance to TKI therapy in patients with NSCLC (12). Patients that harbor a KRAS mutation and EGFR mutations in exons 21 and 20 are extremely rare.

\section{Case report}

A 69-year-old man that had smoked 10 cigarettes/day for 20 years was admitted to The First Affiliated Hospital of Sun Yat-Sen University (Guangzhou, Guangdong, China) in September 2011, due to the presence of an abnormal shadow in the right upper lung that was identified on chest X-ray. Physical examination revealed no significant abnormalities. Chest computed tomography $(\mathrm{CT})$ revealed a 28x36 mm tumor in the upper lobe of the right lung, which was classified as tumor-node-metastasis (TNM) stage T3N0M1. Bronchoscopy revealed a tumor occluding the right upper bronchus, with active bleeding. In order to relieve the respiratory symptoms that resulted from hemoptysis, the patient underwent lobectomy of the right upper lung and mediastinal lymph node dissection. The resected tumor exhibited typical adenocarcinomatous characteristics on hematoxylin and eosin staining. Immunohistochemical (IHC) staining demonstrated strong expression of the transcription factor-1 (Abbott, USA), 

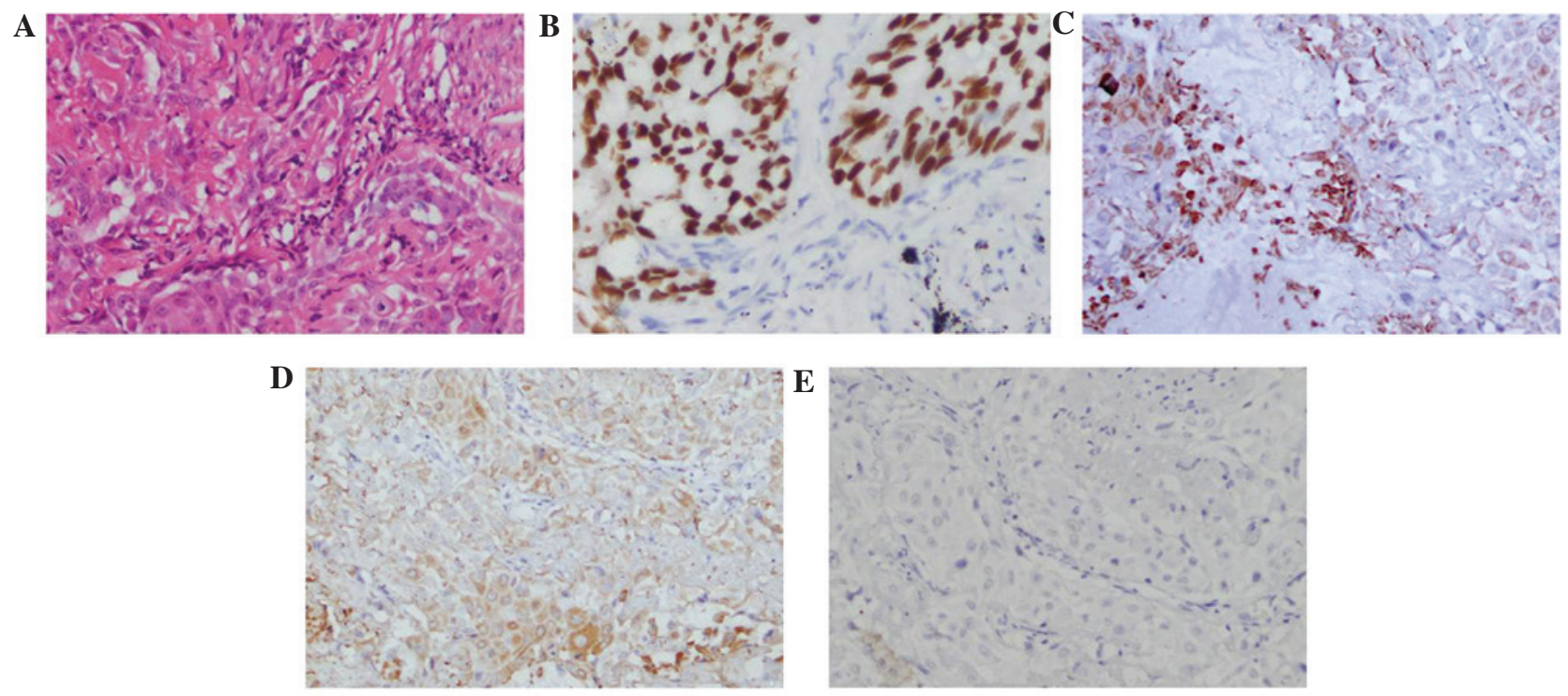

Figure 1. Histological morphology of the primary lung adenocarcinoma, demonstrating a predominantly solid growth pattern. (A) Hematoxilin and eosin staining (magnification, x20). Immunohistochemical analysis revealed the adenocarcinoma to be (B) thyroid transcription factor 1-positive (magnification, x20), (C) carcinoembryonic antigen-positive (magnification, x20), (D) cytokeratin 7-positive (magnification, x20) and (E) p63-negative (magnification, x20).
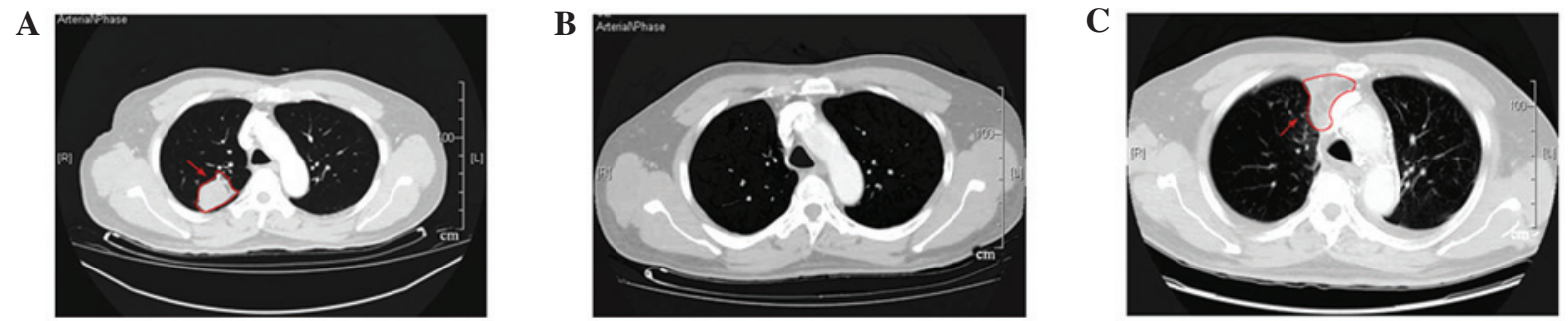

Figure 2. Chest CT scans in a patient with lung adenocarcinoma, revealing the primary tumor (red arrows). (A) Chest CT prior to the administration of gefitinib. (B) Repeated CT at 20 days subsequent to gefitinib treatment. (C) CT revealing disease progression 3 months following gefitinib treatment. CT, computed tomography.

carcinoembryonic antigen (CEA; Dako, Glostrup, Denmark) and cytokeratin-7 (Dako) proteins. The findings of laboratory examinations were within the normal range, with the exception of CEA, which demonstrated a serum level of $6.24 \mathrm{ng} / \mathrm{ml}$ (normal range, $0.00-5.00 \mathrm{ng} / \mathrm{ml}$ ). The pathological diagnosis of the tumor specimen was acinar adenocarcinoma (Fig. 1) harboring one EGFR mutation in exon 21, the L858R mutation. Subsequent to consideration of the poor condition of the patient, treatment with gefitinib was selected at a dose of $250 \mathrm{mg} /$ day for 3 months.

A chest X-ray performed 20 days later revealed that the completely occluded left main bronchus had reopened and that the right lung atelectasis was considerably improved. There were no severe side-effects, such as lung toxicity. However, 3 months following the initiation of gefitinib therapy, the tumor began to enlarge (Fig. 2). Gefitinib therapy was discontinued in order to introduce second-line chemotherapy, consisting of docetaxel $\left(75 \mathrm{mg} / \mathrm{m}^{2}\right.$, once) and gefitinib $(250 \mathrm{mg} /$ day $)$ for 3 weeks, which induced infusion reaction. No notable response was observed. Following discontinuation of the second-line chemotherapy, tumor growth induced empyema and the general condition of the patient gradually deteriorated. Trans-bronchial lung biopsy of the right lung was then performed to perform EGFR and KRAS mutation analysis using amplification refractory mutation system (Amoy Diagnostics Co., Ltd., Haicang, Xiamen, China). In the tumor DNA, the point mutations L858R, in which leucine at amino acid 858 is replaced by arginine, and T790M, in which threonine at amino acid 790 is replaced by methionine, were detected in the EGFR gene, in addition to the KRAS G12V mutation (Fig. 3). However, these findings could not be applied to the treatment strategy as the patient succumbed to respiratory failure 2 months later. The present study was approved by the ethics committee of Sun Yat-Sen University and written informed consent was obtained from the patient's family.

\section{Discussion}

It has been demonstrated that the majority of patients with EGFR TKI-sensitive lung cancer harbor activating mutations in the TK domain of EGFR (13-15), which supports the hypothesis that identifying genetic signatures associated with oncogenic alterations may yield predictive biomarkers for corresponding molecular target inhibitors. Compared to smokers, EGFR mutations have consistently been found to be more common in never-smokers (16). It has been demonstrated that the majority of tumors with activating EGFR mutations present with a non-squamous histology. Retrospective data reported 
A

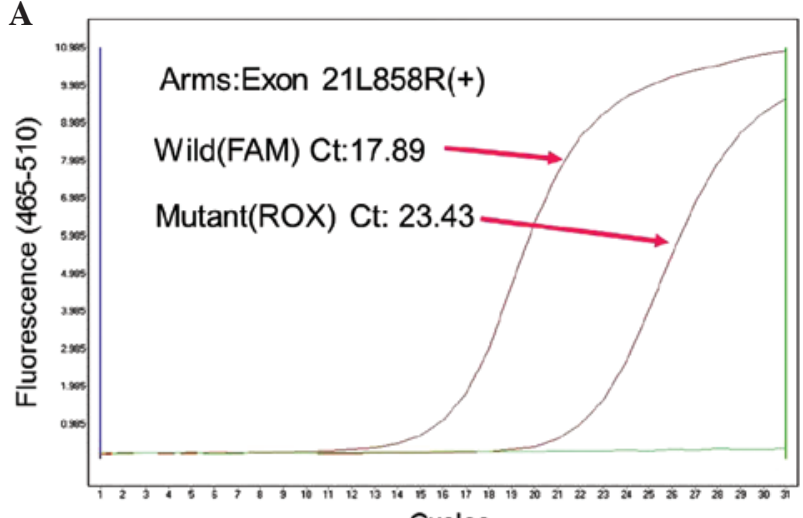

C

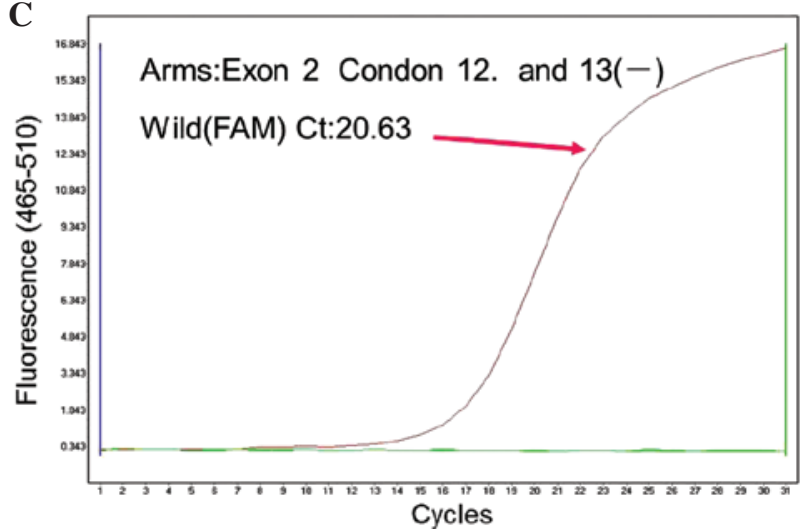

B

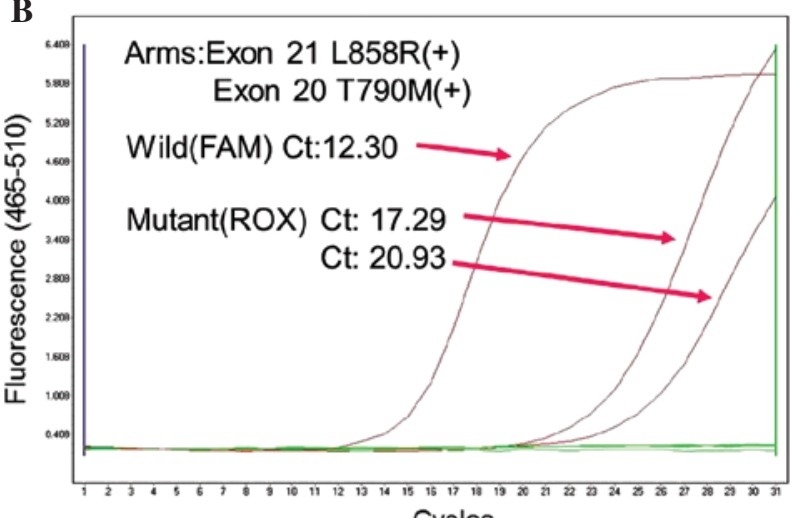

D

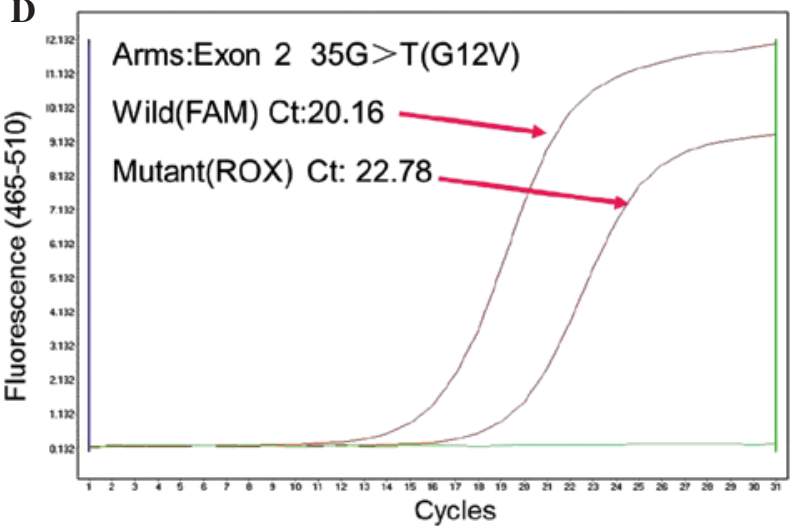

Figure 3. EGFR and KRAS mutations were identified using the ARMS method. (A) EGFR L858R point mutation detected prior to gefitinib treatment. (B) EGFR L858R and T790M point mutations detected subsequent to gefitinib treatment. (C) Absence of KRAS point mutation prior to gefitinib treatment. (D) KRAS 35G $>$ T (G12V) point mutation detected subsequent to gefitinib treatment. EGFR, epidermal growth factor receptor; KRAS, kirsten rat sarcoma viral oncogene homolog; ARMS, amplification-refractory mutation system.

by Tanaka et al reveals that 3-8\% of patients with NSCLC that demonstrated squamous histology present in activating EGFR mutations (17). The present diagnosis of squamous cell carcinoma or adenocarcinoma was based on histomorphological analysis, in cases where the appearance was characteristic, or IHC staining, which was performed using antibodies against TTF-1, p63, CEA and CK. Nuclear expression of TTF-1, identified by IHC, demonstrated the lesion in the present patient to be of primary pulmonary origin. Furthermore, tumor cells were CEA-positive and p63-negative, indicating that they may originate from the glandular epithelium.

Deletion mutations in exon 19 that affect the conserved LREA motif and a single amino acid substitution, leucine to arginine, at codon 858 in exon 21 are associated with increased sensitivity to EGFR TKIs, including gefitinib and erlotinib (18-20). The clinical observation that the present patient with a L858R mutation achieved radiographic tumor regression may be indicative that patients with EGFR L858R-bearing tumors can benefit from gefitinib at the usual clinical doses. However, in a previous study $>20 \%$ of patients demonstrated resistance to EGFR TKIs and all tumors ultimately developed resistance following the initial response (21). The most common molecular mechanism of acquired resistance is a mutation at a second site in the EGFR tyrosine kinase domain, T790M. This mutation confers resistance by increasing the affinity for ATP, with which inhibitors must compete for binding, and also by modestly decreasing the intrinsic affinity for TKIs (22).
In the present study, 3 months subsequent to the initiation of gefitinib therapy, the tumor relapsed and a CT scan demonstrated progressive disease. Molecular analysis demonstrated the presence of an adenocarcinoma harboring two different EGFR mutations, consisting of a mutation in exon 20 (T790M) and a mutation in exon 21 (L858R). Consistent with the role of KRAS mutations as driver mutations, KRAS mutations do not occur in association with EGFR mutations (23-26), although rare exceptions do occur (27). However, the present study reported the case of a patient that possessed tumor DNA harboring KRAS and EGFR mutations, a phenomenon that has been rarely reported. Furthermore, a meta-analysis has revealed that tumors harboring a KRAS mutation are resistant to EGFR TKIs (28), as KRAS mutations lead to constitutive activation of pathways downstream of EGFR. The aforementioned data demonstrated that the recurrence and rapid resistance to gefitinib observed in the present patient may be attributed to the T790M mutation in EGFR and the KRAS mutation simultaneously. In particular, it has been demonstrated in colorectal cancer of an advanced stage that under conditions of EGFR-blockade, pre-existing undetected KRAS mutant cells contribute to relapse $(29,30)$. There are several reasons to expect that KRAS may play a similar role in the acquired resistance to EGFR blockade in lung adenocarcinoma. First, KRAS mutant subpopulations have been identified in lung adenocarcinoma tumors (31). Secondly, in lung adenocarcinoma lesions, KRAS mutation has been reported to confer primary resistance to EGFR blockade (9). 
Thus, the exploration of a more sensitive detecting system for tumor tissues is urgently required in order to provide more reasonable treatment options for patients.

Resistance to targeted therapies is an increasingly reported issue, into which genomic analyses may provide important mechanistic insights for future rational therapeutic approaches. The present study reported a rare case of lung cancer harboring a KRAS mutation and a compound T790M EGFR substitution mutation, which was identified subsequent to the administration of gefitinib treatment to the patient. Thus, to improve the guidance for optimal treatment schemes in individual patients, additional studies that aim to identify the genetic causes of drug resistance at various points during the clinical course are required.

\section{Acknowledgements}

The present study received financial support from the National Natural Science Foundation of China (grant nos., 30900650/H1615, 81372501/H1615, 81172232/H1615 and 81172564/H1625), Guangdong Natural Science Foundation (grant nos., S2012010008378 and S2013010015327), Research Fund for the Doctoral Program of Higher Education of China (grant no., 20120171120086) and Science and Technology Planning Project of Guangdong Province (grant no., 2012B061700078).

\section{References}

1. Gaughan EM and Costa DB: Genotype-driven therapies for non-small cell lung cancer: Focus on EGFR, KRAS and ALK gene abnormalities. Ther Adv Med Oncol 3: 113-125, 2011.

2. Rosell R, Carcereny E, Gervais R, et al; Spanish Lung Cancer Group in collaboration with Groupe Français de Pneumo-Cancérologie and Associazione Italiana Oncologia Toracica: Erlotinib versus standard chemotherapy as first-line treatment for European patients with advanced EGFR mutation-positive non-small-cell lung cancer (EURTAC) A multicentre, open-label, randomised phase 3 trial. Lancet Oncol 13: 239-246, 2012

3. Takano T, Ohe Y, Sakamoto H, et al: Epidermal growth factor receptor gene mutations and increased copy numbers predict gefitinib sensitivity in patients with recurrent non-small-cell lung cancer. J Clin Oncol 23: 6829-6837, 2005.

4. Kobayashi S, Boggon TJ, Dayaram T, et al: EGFR mutation and resistance of non-small-cell lung cancer to gefitinib. N Engl J Med 352: 786-792, 2005.

5. Kosaka T, Yatabe Y, Endoh H, et al: Analysis of epidermal growth factor receptor gene mutation in patients with non-smal cell lung cancer and acquired resistance to gefitinib. Clin Cancer Res 12: 5764-5769, 2006.

6. Riely GJ, Kris MG, Rosenbaum D, et al: Frequency and distinctive spectrum of KRAS mutations in never smokers with lung adenocarcinoma. Clin Cancer Res 14: 5731-5734, 2008.

7. De Luca A and Normanno N: Predictive biomarkers to tyrosine kinase inhibitors for the epidermal growth factor receptor in non-small-cell lung cancer. Curr Drug Targets 11: 851-864, 2010.

8. Mao C, Qiu LX, Liao RY, et al: KRAS mutations and resistance to EGFR-TKIs treatment in patients with non-small cell lung cancer: A meta-analysis of 22 studies. Lung Cancer 69: 272-278, 2010.

9. Pao W, Wang TY, Riely GJ, et al: KRAS mutations and primary resistance of lung adenocarcinomas to gefitinib or erlotinib. PLoS Med 2: e17, 2005.
10. Stella GM, Scabini R, Inghilleri S, et al: EGFR and KRAS mutational profiling in fresh non-small cell lung cancer (NSCLC) cells. J Cancer Res Clin Oncol 139: 1327-1335, 2013.

11. Pennycuick A, Simpson T, Crawley D, et al: Routine EGFR and KRAS Mutation analysis using COLD-PCR in non-small cell lung cancer. Int J Clin Pract 66: 748-752, 2012.

12. Hammerman PS, Jänne PA and Johnson BE: Resistance to epidermal growth factor receptor tyrosine kinase inhibitors in non-small cell lung cancer. Clin Cancer Res 15: 7502-7509, 2009.

13. Lynch TJ, Bell DW, Sordella R, et al: Activating mutations in the epidermal growth factor receptor underlying responsiveness of non-small-cell lung cancer to gefitinib. N Engl J Med 350: 2129-2139, 2004

14. Sequist LV, Martins RG, Spigel D, et al: First-line gefitinib in patients with advanced non-small-cell lung cancer harboring somatic EGFR mutations. J Clin Oncol 26: 2442-2449, 2008.

15. Paez JG, Jänne PA, Lee JC, et al: EGFR mutations in lung cancer: Correlation with clinical response to gefitinib therapy. Science 304: 1497-1500, 2004

16. Cortes-Funes H, Gomez C, Rosell R, et al: Epidermal growth factor receptor activating mutations in Spanish gefitinib-treated non-small-cell lung cancer patients. Ann Oncol 16: 1081-1086, 2005.

17. Tanaka T, Matsuoka M, Sutani A, et al: Frequency of and variables associated with the EGFR mutation and its subtypes. Int J Cancer 126: 651-655, 2010.

18. Mitsudomi T, Morita S, Yatabe Y, et al; West Japan Oncology Group: Gefitinib versus cisplatin plus docetaxel in patients with non-small-cell lung cancer harbouring mutations of the epidermal growth factor receptor (WJTOG3405): An open label, randomised phase 3 trial. Lancet Oncol 11: 121-128, 2010.

19. Mok TS, Wu YL, Thongprasert S, et al: Gefitinib or carboplatin-paclitaxel in pulmonary adenocarcinoma. N Engl J Med 361: 947-957, 2009.

20. Rosell R, Viteri S, Molina MA, et al: Epidermal growth factor receptor tyrosine kinase inhibitors as first-line treatment in advanced nonsmall-cell lung cancer. Curr Opin Oncol 22: $112-120,2010$

21. Pao W and Chmielecki J: Rational, biologically based treatment of EGFR-mutant non-small-cell lung cancer. Nat Rev Cancer 10: 760-774, 2010

22. Yun CH, Mengwasser KE, Toms AV, et al: The T790M mutation in EGFR kinase causes drug resistance by increasing the affinity for ATP. Proc Natl Acad Sci USA 105: 2070-2075, 2008.

23. Yip PY, Yu B, Cooper WA, et al: Patterns of DNA mutations and ALK rearrangement in resected node negative lung adenocarcinoma. J Torac Oncol 8: 408-414, 2013.

24. Ding L, Getz G, Wheeler DA, et al: Somatic mutations affect key pathways in lung adenocarcinoma. Nature 455: 1069-1075, 2008.

25. Shigematsu H, Lin L, Takahashi T, et al: Clinical and biological features associated with epidermal growth factor receptor gene mutations in lung cancers. J Natl Cancer Inst 97: 339-346, 2005.

26. Tam IY, Chung LP, Suen WS, et al: Distinct epidermal growth factor receptor and KRAS mutation patterns in non-small cell lung cancer patients with different tobacco exposure and clinicopathologic features. Clin Cancer Res 12: 1647-1653, 2006.

27. Schmid K, Oehl N, Wrba F, et al: EGFR/KRAS/BRAF mutations in primary lung adenocarcinomas and corresponding locoregional lymph node metastases. Clin Cancer Res 15: 4554-4560, 2009.

28. Linardou H, Dahabreh IJ, Kanaloupiti D, et al: Assessment of somatic k-RAS mutations as a mechanism associated with resistance to EGFR-targeted agents: A systematic review and meta-analysis of studies in advanced non-small-cell lung cancer and metastatic colorectal cancer. Lancet Oncol 9: 962-972, 2008

29. Misale S, Yaeger R, Hobor S, et al: Emergence of KRAS mutations and acquired resistance to anti-EGFR therapy in colorectal cancer. Nature 486: 532-536, 2012.

30. Diaz LA Jr, Williams RT, Wu J, et al: The molecular evolution of acquired resistance to targeted EGFR blockade in colorectal cancers. Nature 486: 537-540, 2012.

31. Parsons BL and Myers MB: Personalized cancer treatment and the myth of KRAS wild-type colon tumors. Discov Med 15: 259-267, 2013. 